\title{
Ferromagnetism and Antiferromagnetism of Correlated Topological Insulator with Flat Band
}

\author{
Jing He, ${ }^{1}$ Bo Wang, ${ }^{1}$ and Su-Peng $\mathrm{Kou}^{1}$, , \\ ${ }^{1}$ Department of Physics, Beijing Normal University, Beijing 100875, China
}

\begin{abstract}
In this paper, based on the mean field approach and random-phase-approximation, we study the magnetic properties of the spinful Haldane model on honeycomb lattice of topological flat band with on-site repulsive Coulomb interaction. We find that the antiferromagnetic (AF) order is more stable than ferromagnetic (FM) order at (or near) half-filling; while away from half-filling the phase diagram becomes complex: At large doping, FM order is more stable than AF order due to the flatness of band structure. In particular, we find that at quarter filling case, the system becomes a $Q=1$ topological insulator which is induced by the FM order.
\end{abstract}

\section{INTRODUCTION}

From 1980 year, topological ordered states become a hot topic and a lot of efforts had been done on this issue [1]. Integer and fractional quantum Hall effect (IQHE and FQHE) have attracted great attention and been studied a great deal since first discovered in two-dimensional electron gas with Laudau levels induced by strong magnetic field [2, 3]. Haldane's honeycomb model[4] demonstrates IQHE in lattice model without Landau levels while FQHE had not been discovered in lattice models until recently 5 -7]. The topologically nontrivial flat-land was pointed to be key in realizing FQHE in lattice models based on the mechanism of quadratic band touching. And the lattice models are designed reaching a high flatness ratio (the ratio of the band gap over bandwidth) about 50 in different lattice models (kagóme lattice, square lattice and honeycomb lattice) [57]. In these models, the hoppings of next-nearest neighbor $(\mathrm{NNN})$ or next-next-nearest neighbor (NNNN) besides nearest neighbor (NN) are introduced to achieve the flat-bands. And the flat-bands of these models are shown to be with non-trivial topology (with non-zero Chern number). To obtain FQHE states, people always consider these tight-binding models of spinless fermions or bosons with the NN and the NNN repulsions under a hard-core condition. Then the numerical results confirm the existence of topologically nontrivial flat-band [8, 9].

On the other hand, the spin orders are important magnetic properties in a strongly correlated electron system, on which people had also paid much attention [10]. For example, an interesting problem is about the ferromagnetic (FM) order in correlated electron system with flat band. According to the Stoner criteria, for correlated electronic system with flat or almost flat band, the Coulomb interaction will remove the band degeneracy and drive the system into a ferromagnetic ground state [11, 12]. So we may ask a question : in a spinful fermionic lattice model of topologically nontrivial flat-band with on-site Coulomb interaction, whether the

\footnotetext{
${ }^{*}$ Corresponding author; Electronic address: spkou@bnu.edu.cn
}

ground state is FM order? This is the starting point of this paper.

After doing mean field calculations, in a typical twocomponent fermionic lattice model of topologically nontrivial flat-band with on-site Coulomb interaction (the spinful Haldane model on honeycomb lattice [4, 15, 16]), we find that the $\mathrm{AF}$ order is more stable than FM order at (or near) half-filling; while away from half-filling the phase diagram becomes complex: At large doping, FM order is more stable than AF order due to the flat band. In particular, we found that at quarter filling case, the system becomes a $Q=1$ topological insulator which is induced by the FM order. In Ref.[13], it was proved that the ground state of the Hubbard model with topological flat-bands is a ferromagnetic order when the lowest flat band is half-filled. Our results are consistent with this rigorous provement. In addition, in Ref. [14], quantum anomalous Hall effect in a flat-band ferromagnet on a two dimensional decorated lattice with spin-orbit coupling was also predicted.

The paper is organized as follows. Firstly, we write down the Hamiltonian of the spinful Haldane model on honeycomb lattice with topological flat band in Sec.II. In Sec.III, we do mean field calculations and get the phase diagram. In Sec.IV, we discuss magnetic properties and topological properties for the the half filling case. Then in Sec.V, we discuss the magnetic properties and the topological properties for the the quarter filling case. Next we discuss the other doping cases in VI. Finally, the conclusions are given in Sec.VII.

\section{THE MODEL HAMILTONIAN}

In this paper we start from the spinful Haldane model with the on-site Coulomb interaction with the Hamilto- 


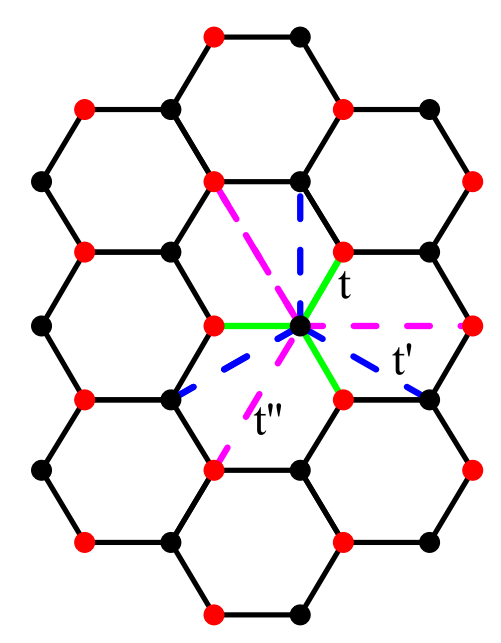

FIG. 1: (Color online) The illustration of the honeycomb lattice. The red and black dots represent the bipartite lattice. The green lines, dash blue lines and dash magenta lines are the nearest hopping, $t$, the next nearest hopping $t^{\prime}$ and the next next nearest hopping, $t^{\prime \prime}$, respectively.

nian as [4, 15, 16]

$$
\begin{aligned}
H & =-t \sum_{\langle i, j\rangle, \sigma} \hat{c}_{i \sigma}^{\dagger} \hat{c}_{j \sigma}-t^{\prime} \sum_{\langle\langle i, j\rangle\rangle, \sigma} e^{i \phi_{i j}} \hat{c}_{i \sigma}^{\dagger} \hat{c}_{j \sigma} \\
& -t^{\prime \prime} \sum_{\langle\langle\langle i, j\rangle\rangle\rangle, \sigma} \hat{c}_{i \sigma}^{\dagger} \hat{c}_{j \sigma}+U \sum_{i} \hat{n}_{i \uparrow} \hat{n}_{i \downarrow} \\
& -\mu \sum_{i, \sigma} \hat{c}_{i \sigma}^{\dagger} \hat{c}_{i \sigma}+h . c .
\end{aligned}
$$

where $U$ is the on-site Coulomb repulsive interaction strength and $\mu$ is the chemical potential. $t, t^{\prime}$ and $t^{\prime \prime}$ are the nearest neighbor, the next nearest neighbor and the next next nearest neighbor hoppings, respectively (See Fig.1). We all know that the honeycomb lattice is a bipartite lattice which is represent by the red and black dots in Fig.1, and the green lines, dash blue lines and dash magenta lines are the nearest hoppings, the next nearest hoppings and the next next nearest hoppings, respectively. In the flat-band limit we have $t^{\prime}=0.6 t$, $t^{\prime \prime}=-0.58 t[7] . \phi_{i j}=0.4 \pi$ is a complex phase to the next nearest neighbor hopping, of which the positive phase is set to be clockwise 7].

For the free electrons case $(U / t=0)$, the lower energy band becomes flat with nonzero Chern numbers $Q=2$ and the flatness ratio is about 50 (See Fig.2). From Fig.2, we can see that (a) is the totally energy bands, spin up and spin down are degenerate due to the spin-rotation symmetry and (b) is the amplification of the flat band. The flat band still has dispersion but the bandwidth is much smaller than the band gap. Fig.3 illustrates the existence of edge states in this $Q=2$ topological insulator. (a)

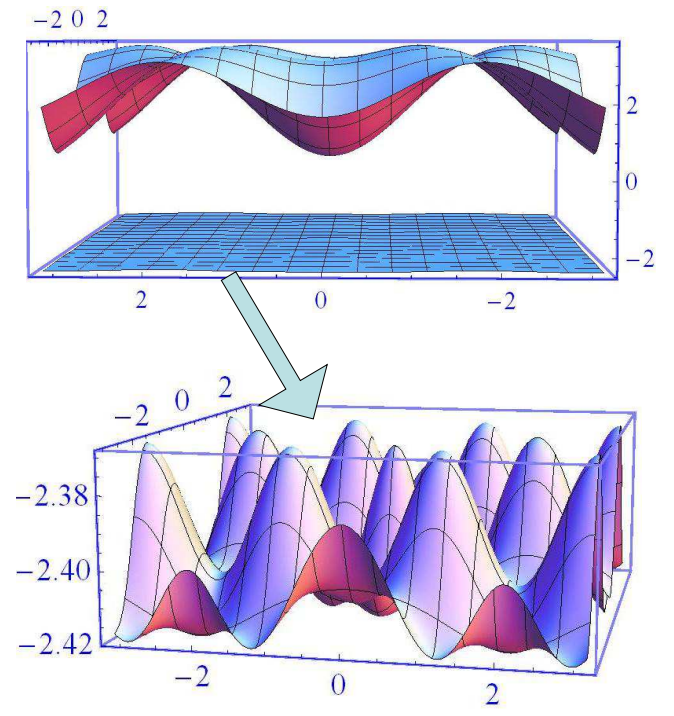

FIG. 2: (Color online) The free electronic dispersion $(U / t=$ 0 ) at $t^{\prime}=0.6 t, t^{\prime \prime}=-0.58 t$ and $\phi_{i j}=0.4 \pi$. (a) is the two bands, of which each band is doubly degenerate. (b) shows the details of the flat band. One can see that the bandwidth is much smaller than the band gap.

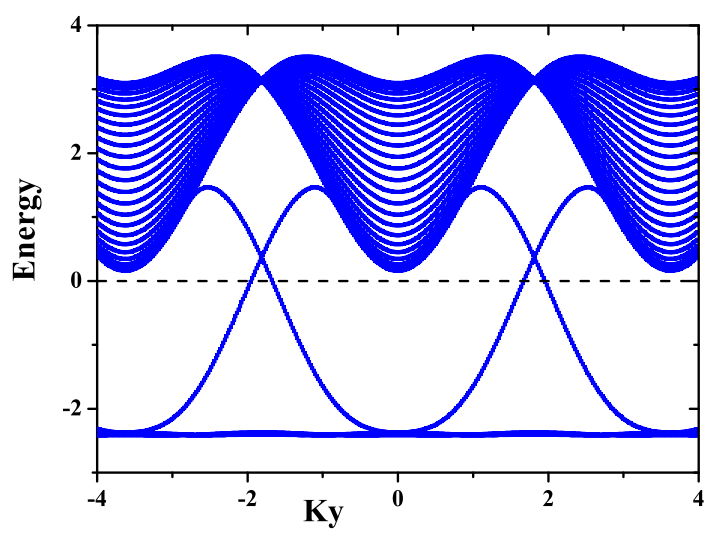

FIG. 3: (Color online) The Zigzag edge states along $\mathrm{x}$ direction at $U / t=0, t^{\prime}=0.6 t, t^{\prime \prime}=-0.58 t$ and $\phi_{i j}=0.4 \pi$. The black dash line is the position of chemical potential.

\section{MEAN FIELD APPROACH}

Next, we consider the interacting case of $U / t \neq 0$. An interesting question is: what is the ground state for the spinful Haldane model with the on-site interaction in the TFB limit when the $U / t \neq 0$ ? As discussed above, people know that for the case of an interacting fermion model with flat-band, the ground state is always a FM order. On the other hand, near the half filling, the superexchange effect will lead to an antiferromagnetic (AF) spin interaction between two local spin moments nearby. 
Thus to answer this question, we will study the competition between AF order and FM order by the following mean-field ansatzs :

$$
\left\langle\hat{c}_{i, \sigma}^{\dagger} \hat{c}_{i, \sigma}\right\rangle=\frac{1}{2}\left[n+(-1)^{i} \sigma M_{A F}\right]
$$

and

$$
\left\langle\hat{c}_{i, \sigma}^{\dagger} \hat{c}_{i, \sigma}\right\rangle=\frac{1}{2}\left(n+\sigma M_{F}\right)
$$

where $M_{A F}$ is the staggered antiferromagnetic order parameter and $M_{F}$ is the ferromagnetic order parameter, $n$ is the average particle number on each site. For the case of spin-up and spin-down, we have $\sigma=1$ and $\sigma=-1$, respectively.

\section{A. AF order}

Firstly we consider the instability of AF order. For the AF order, we have $M_{A F} \neq 0$, now the effective Hamilto- nian can be written as:

$$
\begin{aligned}
H & =-t \sum_{\langle i, j\rangle, \sigma} \hat{c}_{i \sigma}^{\dagger} \hat{c}_{j \sigma}-t^{\prime} \sum_{\langle\langle i, j\rangle\rangle, \sigma} e^{i \phi_{i j}} \hat{c}_{i \sigma}^{\dagger} \hat{c}_{j \sigma}-t^{\prime \prime} \sum_{\langle\langle\langle i, j\rangle\rangle\rangle, \sigma} \hat{c}_{i \sigma}^{\dagger} \hat{c}_{j \sigma} \\
& -\sum_{i}(-1)^{i} \Delta_{A} \hat{c}_{i}^{\dagger} \sigma_{z} \hat{c}_{i}+\sum_{i, \sigma}\left(\frac{U n}{2}-\mu\right) \hat{c}_{i \sigma}^{\dagger} \hat{c}_{i \sigma}+\text { h.c. }
\end{aligned}
$$

where $\Delta_{A}=U M_{A F} / 2$ and $\sigma_{z}$ is Pauli matrix. $n=$ $1-d$ is the average particle number on each site and $d$ is the hole concentration. After Fourier transformation, the Hamiltonian in momentum space becomes

$$
H=\sum_{k} \Psi_{k}^{\dagger} h_{A k} \Psi_{k}
$$

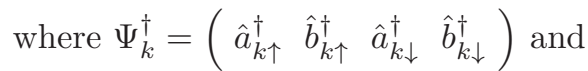

$$
h_{A k}=\left(\begin{array}{cccc}
-t^{\prime}(C-D)+\Delta_{A} & \xi_{k} & 0 & 0 \\
\xi_{k}^{*} & -t^{\prime}(C+D)-\Delta_{A} & 0 & 0 \\
0 & 0 & -t^{\prime}(C-D)-\Delta_{A} & \xi_{k} \\
0 & 0 & \xi_{k}^{*} & -t^{\prime}(C+D)+\Delta_{A}
\end{array}\right)
$$

where $\xi_{k}=-t(A+i B)-t^{\prime \prime}(E+i F)$.

Then we can get the spectrum of the electrons as

$$
\begin{aligned}
& E_{A, k, 1, \pm}=-t^{\prime} C+\sqrt{\left|\xi_{k}\right|^{2}+\left(t^{\prime} D \pm \Delta_{A}\right)^{2}} \\
& E_{A, k, 2, \pm}=-t^{\prime} C-\sqrt{\left|\xi_{k}\right|^{2}+\left(t^{\prime} D \pm \Delta_{A}\right)^{2}}
\end{aligned}
$$

where

$$
\begin{aligned}
& A=2 \cos \left(k_{x} / 2\right) \cos \left(\sqrt{3} k_{y} / 2\right)+\cos k_{x} \\
& B=2 \sin \left(k_{x} / 2\right) \cos \left(\sqrt{3} k_{y} / 2\right)-\sin k_{x} \\
& C=2 \cos \phi \sum_{i=1}^{3} \cos \left(\mathbf{k} \cdot \mathbf{b}_{i}\right) \\
& D=2 \sin \phi \sum_{i=1}^{3} \sin \left(\mathbf{k} \cdot \mathbf{b}_{i}\right) \\
& E=2 \cos k_{x} \cos \left(\sqrt{3} k_{y}\right)+\cos 2 k_{x} \\
& F=-2 \sin k_{x} \cos \left(\sqrt{3} k_{y}\right)+\sin 2 k_{x}
\end{aligned}
$$

where $\mathbf{b}_{i}$ is the next nearest neighbor vectors.
At different doping, by minimizing the free energy of the mean field, the self-consistent equations at zero temperature in the reduced BZ are 17, 18]:

$$
\begin{aligned}
1 & =\frac{1}{N_{s} M_{A F}}\left[\sum_{E_{A, k, 2,+}<\mu_{e f f}} \frac{t^{\prime} D+\Delta_{A}}{2 \xi_{k 1}}\right. \\
& \left.-\sum_{E_{A, k, 2,-}<\mu_{e f f}} \frac{t^{\prime} D-\Delta_{A}}{2 \xi_{k 2}}\right], \\
1-d & =\frac{1}{2 N_{s}}\left[\sum_{E_{A, k, 2,+}<\mu_{e f f}} 1+\sum_{E_{A, k, 2,-}<\mu_{e f f}} 1\right]
\end{aligned}
$$

where $\mu_{\text {eff }}=\mu-\frac{U n}{2}$ and $N_{s}$ is the number of the unit cells and

$$
\begin{aligned}
& \xi_{k 1}=\sqrt{\left|\xi_{k}\right|^{2}+\left(t^{\prime} D+\Delta_{A}\right)^{2}} \\
& \xi_{k 2}=\sqrt{\left|\xi_{k}\right|^{2}+\left(t^{\prime} D-\Delta_{A}\right)^{2}}
\end{aligned}
$$

In Fig.4, we give the electronic dispersions for AF order at $U / t=7.0$ for half-filling. Comparing with Fig.2 and Fig.4, we can see that AF order parameter can change the flat band into a dispersive one. 


\section{B. FM order}

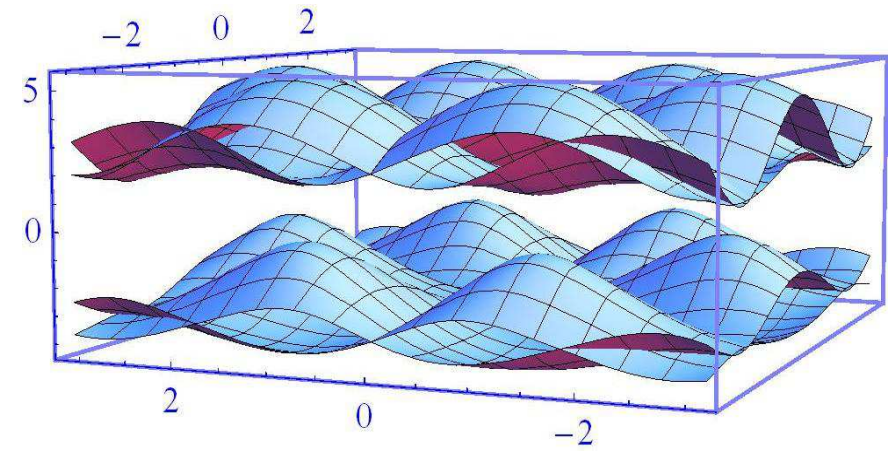

FIG. 4: (Color online) The electronic dispersions for AF order at $U / t=7.0, t^{\prime}=0.6 t, t^{\prime \prime}=-0.58 t$ and $\phi_{i j}=0.4 \pi$ for half filling. We can see that the AF order parameter changes the flat band into a dispersive one.
Secondly we consider the instability of FM order. For FM order, we have $M_{F} \neq 0$, now the effective Hamiltonian can be written as

$$
\begin{aligned}
H & =-t \sum_{\langle i, j\rangle, \sigma} \hat{c}_{i \sigma}^{\dagger} \hat{c}_{j \sigma}-t^{\prime} \sum_{\langle\langle i, j\rangle\rangle, \sigma} e^{i \phi_{i j}} \hat{c}_{i \sigma}^{\dagger} \hat{c}_{j \sigma}-t^{\prime \prime} \sum_{\langle\langle\langle i, j\rangle\rangle\rangle, \sigma} \hat{c}_{i \sigma}^{\dagger} \hat{c}_{j \sigma} \\
& -\sum_{i} \Delta_{F} \hat{c}_{i}^{\dagger} \sigma_{z} \hat{c}_{i}+\sum_{i, \sigma}\left(\frac{U n}{2}-\mu\right) \hat{c}_{i \sigma}^{\dagger} \hat{c}_{i \sigma}+\text { h.c. }
\end{aligned}
$$

where $\Delta_{F}=U M_{F} / 2$. After Fourier transformation, the Hamiltonian in momentum space becomes

$$
H=\sum_{k} \Psi_{k}^{\dagger} h_{F k} \Psi_{k}
$$

where

$$
h_{F k}=\left(\begin{array}{cccc}
-t^{\prime}(C-D)-\Delta_{F} & \xi_{k} & 0 & 0 \\
\xi_{k}^{*} & -t^{\prime}(C+D)-\Delta_{F} & 0 & 0 \\
0 & 0 & -t^{\prime}(C-D)+\Delta_{F} & \xi_{k} \\
0 & 0 & \xi_{k}^{*} & -t^{\prime}(C+D)+\Delta_{F}
\end{array}\right)
$$

Then we can get the spectrum of the electrons in FM order as

$$
\begin{aligned}
& E_{F, k, 1, \pm}=-t^{\prime} C-\Delta_{F} \pm \sqrt{\left|\xi_{k}\right|^{2}+\left(t^{\prime} D\right)^{2}} \\
& E_{F, k, 2, \pm}=-t^{\prime} C+\Delta_{F} \pm \sqrt{\left|\xi_{k}\right|^{2}+\left(t^{\prime} D\right)^{2}}
\end{aligned}
$$

At different doping, by minimizing the free energy of the mean field, the self-consistent equations at zero temperature in the reduced $\mathrm{BZ}$ are

$$
\begin{aligned}
1 & =\frac{1}{N_{s} M_{F}}\left[\sum_{E_{F, k, 1,-}<\mu_{e f f}} \frac{1}{2}\right. \\
& \left.+\sum_{E_{F, k, 1,+}<\mu_{e f f}} \frac{1}{2}-\sum_{E_{F, k, 2,-}<\mu_{e f f}} \frac{1}{2}\right] \\
1-d & =\frac{1}{2 N_{s}}\left[\sum_{E_{F, k, 1,-}<\mu_{e f f}} 1\right. \\
& \left.+\sum_{E_{F, k, 1,+}<\mu_{e f f}} 1+\sum_{E_{F, k, 2,-}<\mu_{e f f}} 1\right]
\end{aligned}
$$

where $\mu_{\text {eff }}=\mu-\frac{U n}{2}$ and $N_{s}$ is the number of the unit cells.
In Fig.5, we give the electronic dispersions for FM order at $U / t=7.0$ for half-filling. Comparing with Fig.2 and Fig.5, we can see that FM order parameter only splits the energy of the flat band without changing the flatness ratio.

\section{Global phase diagram}

Then by using mean field approach above we can get the global phase diagram for different doping in Fig.6 when the parameters are $t^{\prime}=0.6 t, t^{\prime \prime}=-0.58 t$, $\phi_{i j}=0.4 \pi$. From Fig.6, we can see that there exist seven phases: normal metal, FM-metal, AF-metal, $Q=1 \mathrm{FM}$ ordered topological Insulator, $Q=2$ topological insulator with quantized anomalous Hall effect (we may also call it QAH state), A-type antiferromagnetic-spin density wave (A-type AF-SDW) topological insulator and trivial AF order.

At half filling case $d=0$, in the weakly coupling limit $(U / t<5.932)$, the ground state is a $Q=2$ topological insulator with flat-band. In the strong coupling region $(U / t>5.932)$, due to $M_{A F} \neq 0$, the ground state becomes an AF-SDW insulator. And at half filling case, the AF order always has lower energy than FM order. When after doping, the system is away from half filling, 


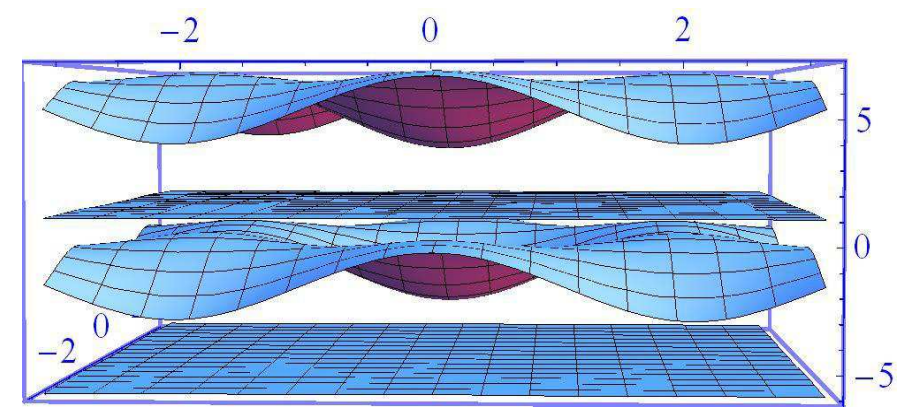

FIG. 5: (Color online) The electronic dispersions for FM order at $U / t=7.0, t^{\prime}=0.6 t, t^{\prime \prime}=-0.58 t$ and $\phi_{i j}=0.4 \pi$ for half filling. We can see that FM order parameter splits the two degenerate flat bands without changing their flat ratio.

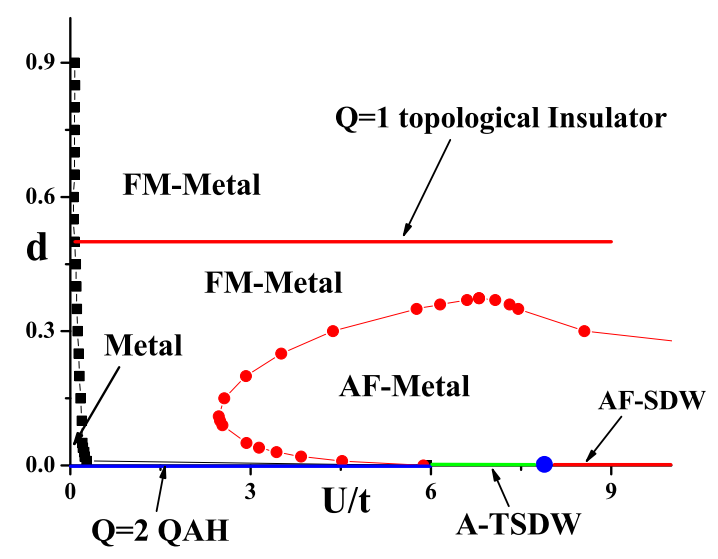

FIG. 6: (Color online) The global phase diagram for the parameters to be $t^{\prime}=0.6 t, t^{\prime \prime}=-0.58 t$ and $\phi_{i j}=0.4 \pi$. At half filling case $d=0$, there are three phases: $Q=2$ topological insulator with quantized anomalous Hall effect, A-TSDW and trivial AF order. For the quarter filling case: $d=0.5$, there are two phases : paramagnetic Metal, $Q=1$ topological Insulator with FM order. For other cases, there are three phases for the region of $0<d<0.5$ : paramagnetic Metal, FM-metal and AF-metal while there are two phases for the region of $0.5<d<1$ : paramagnetic Metal and FM-metal.

$d \neq 0$. Now the repulsive interaction is highly relevant for a fermion system with flat-band. And small interaction will lead to an FM order. Thus in the phase diagram, away from half filling $(d \neq 0)$, there exists a quantum phase transition between paramagnetic metallic phase and FM order for little interaction strength due to a big flatness ratio. And we find a narrow paramagnetic metallic phase. At hole concentration $d=0.5$, the

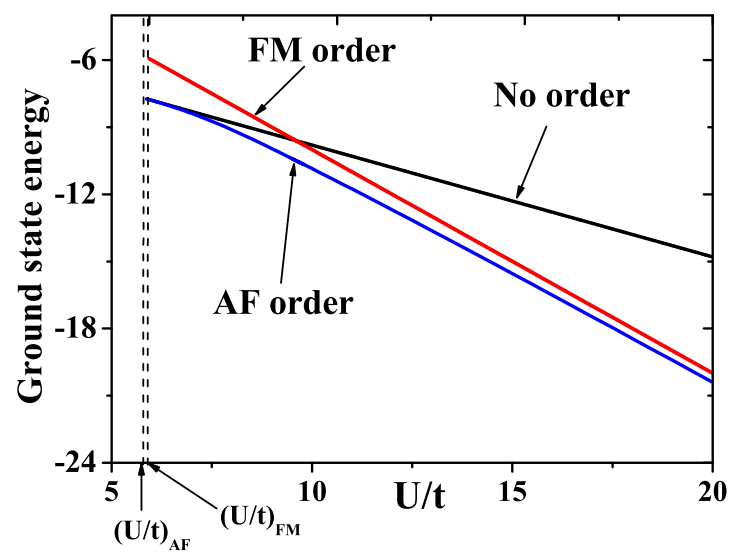

FIG. 7: (Color online) The ground state energies of every site for AF order (Green line), FM order (Red line) and on spin order (black) per site at half filling for the parameters as $t^{\prime}=0.6 t, t^{\prime \prime}=-0.58 t \phi_{i j}=0.4 \pi$.

situation becomes much different : the FM order is really a $Q=1$ topological insulator. For other hole concentration cases $d \neq 0.5, d \neq 1$, the ground state becomes a FM metal. When the interaction strength becomes bigger, FM order is stable in a large region in the global phase diagram due to the flatness of the fermion dispersion. Only near half filling, the ground state energy for every site of AF order may be lower than FM order at some intermediate interaction strength (See below). And there exist first order quantum phase transitions between FM order and AF order (See the red dot line in Fig.6).

In the following, we will give a detailed discussion on the cases of $d=0, d=0.5$ and $d \neq 0,0.5$, respectively.

\section{HALF FILLING CASE : $d=0$}

Firstly we consider the half filling case. At half filling $d=0$, the particle number on average site is $n=1$ and the chemical potential $\mu=U / 2\left(\mu_{\text {eff }}=0\right)$. From Fig.6, we can see that at $d=0$ the ground state is always dominated by the AF-SDW, for the ground state energy of AF order per site is lower than the FM order (See Fig.7). In the weakly interaction limit $(U / t<5.932)$, the ground state is a $Q=2$ topological insulator with flat-band. In the strong interaction region $(U / t>5.932)$, due to $M_{A F} \neq 0$, the ground state becomes an AF-SDW insulator.

Next we plot a phase diagram at half filling with different values of $t^{\prime \prime} / t$ in Fig.8 (the purple dash line denotes $t^{\prime \prime}=-0.58 t$, the dispersion of largest flat ratio). In Fig.8, there are three quantum phase transitions $(U / t)_{F M},(U / t)_{A F}$, and a topological quantum phase transition (the red line) that divide four phases: $Q=2$ topological insulator, FM order, A-type topological AF-SDW (A-TSDW) and trivial AF order. For the 


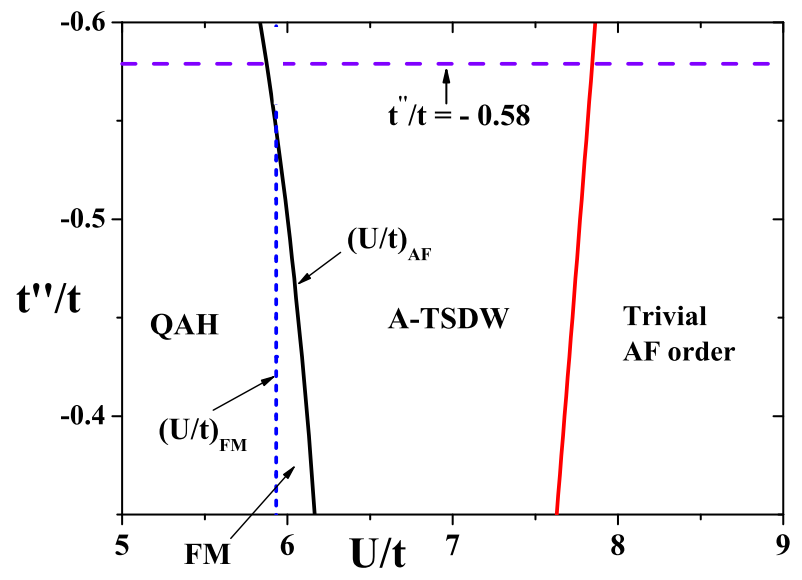

FIG. 8: (Color online) The phase diagram at half filling: there are four phases, QAH, FM-SDW, A-TSDW and Trivial AF order. The dash blue line represent the FM phase transition $(U / t)_{F M}$, the black line represent the AF phase transition $(U / t)_{A F}$, the red line represent a topological quantum phase transition. The purple dash line is the case with largest flat ratio, $t^{\prime}=0.6 t, t^{\prime \prime}=-0.58 t, \phi_{i j}=0.4 \pi$.

flat-band case (the purple dash line of $t^{\prime \prime}=-0.58 t$ ), one can see that the $Q=2$ topological insulator occurs in the weak interaction, and with the increasing of $U / t$, firstly the system turns into A-TSDW state with $M_{A F} \neq 0$, and then turns into the AF insulator with trivial topological properties. Now there only exist two types of phase transitions: one is the magnetic phase transition (the black line in Fig.8) between a magnetic order state with $M_{A F} \neq 0$ and a non-magnetic state with $M_{A F}=0$, the other is a topological quantum phase transition (the red line in Fig.8) which is characterized by the condition of zero fermion energy gaps (see the blue dot in Fig.6). And in Fig.9 and Fig.10, we plot the staggered magnetization and the energy gap for the case with the parameters $t^{\prime}=0.6 t, t^{\prime \prime}=-0.58 t$ and $\phi_{i j}=0.4 \pi$ at half filling.

In Fig.8, there is a dash blue line which is the boundary between $Q=2$ topological insulator and FM order. At half filling case, the ground state energy of AF order per site is lower than the FM order. While away from $t^{\prime \prime}=-0.58 t$ (the dispersion of largest flat ratio), there may still exist a narrow window of an FM order for $t^{\prime \prime} / t>$ -0.55 .

At the half filling case, when the AF order parameter is non-zero, the electronic band structure will be reorganized. Thus in AF order, the spectrums of electrons are dispersive. In addition, we go beyond the mean field theory to study the collective spin fluctuations by random phase approximation (RPA) method. We obtain the dispersion of spin excitations for the ground state. See Fig.11. From Fig.11, one can see that in the AF or-

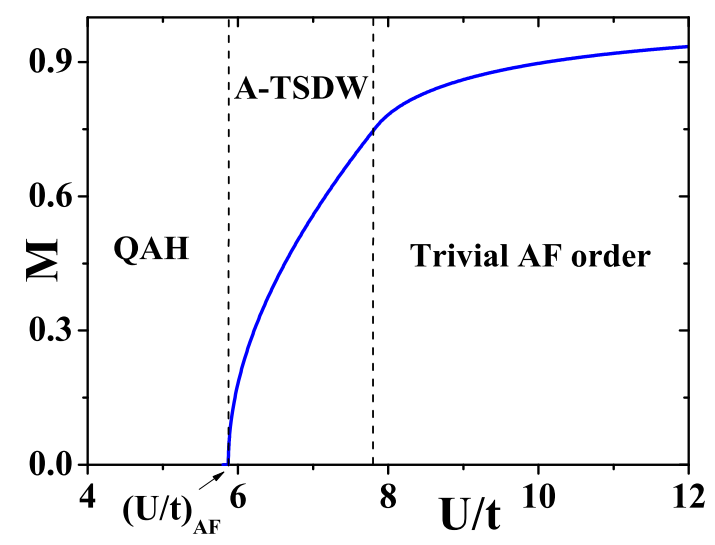

FIG. 9: (Color online) The staggered magnetization $M_{A F}$ for the half filling case with the parameters $t^{\prime}=0.6 t, t^{\prime \prime}=-0.58 t$ and $\phi_{i j}=0.4 \pi$. There are two quantum phase transitions (the black dash lines) : one is the magnetic phase transition at $(U / t)_{A F}$, the other is a topological quantum phase transition with gap closing.

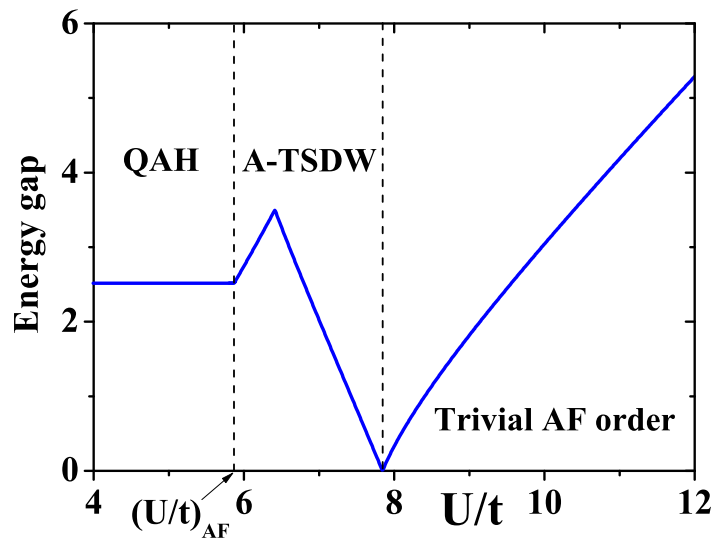

FIG. 10: (Color online) The energy gap of electrons for the half filling case with the parameters to be $t^{\prime}=0.6 t$, $t^{\prime \prime}=-0.58 t$ and $\phi_{i j}=0.4 \pi$. There are two quantum phase transitions (the black dash lines) : one is the magnetic phase transition at $(U / t)_{A F}$, the other is a topological quantum phase transition with gap closing.

der where $U / t>5.932,(U / t=9.0$ in trivial AF-SDW order and $U / t=7.0$ in A-TSDW order) there exist gapless Goldstone modes - spin waves. We also calculate the dispersions of the spin wave of the AF order away from the flat-band case, $t^{\prime}=0.6 t, t^{\prime \prime}=-0.54 t, \phi_{i j}=0.4 \pi$ with $U / t=7.0$ (blue line) and $U / t=9.0$ (pink line). From this results, we can say that the spin waves are dispersive and have linear behavior, $\omega(k) \propto q$ around $\Gamma$ pont, and also don't show anomalous behaviors at the flat band case $\left(t^{\prime \prime}=-0.58 t\right)$ in both trivial AF-SDW order and A-TSDW order. 


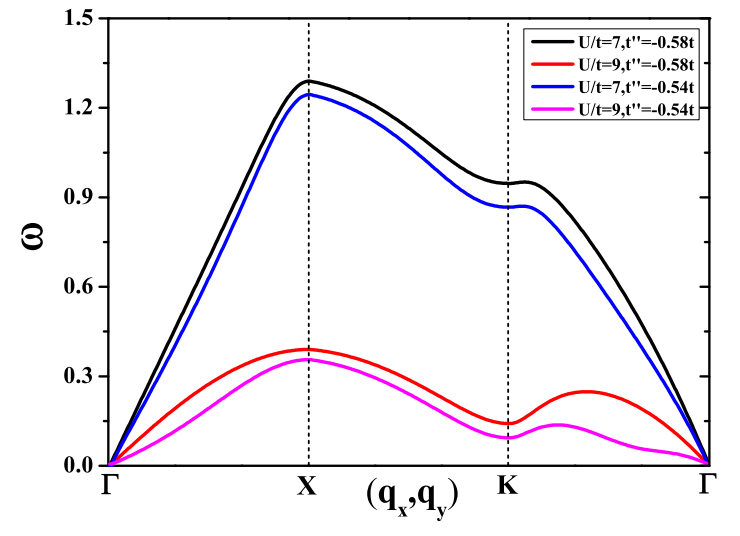

FIG. 11: (Color online) The dispersions of the spin wave of the $\mathrm{AF}$ order for the case of largest flat ratio of the parameters are $t^{\prime}=0.6 t, t^{\prime \prime}=-0.58 t, \phi_{i j}=0.4 \pi$ with $U / t=7.0$ (balck line) and $U / t=9.0$ (red line) at half filling. The dispersions of the spin wave of the AF order away from the flat-band case are also given : $t^{\prime}=0.6 t, t^{\prime \prime}=-0.54 t, \phi_{i j}=0.4 \pi$ with $U / t=7.0$ (blue line) and $U / t=9.0$ (pink line).

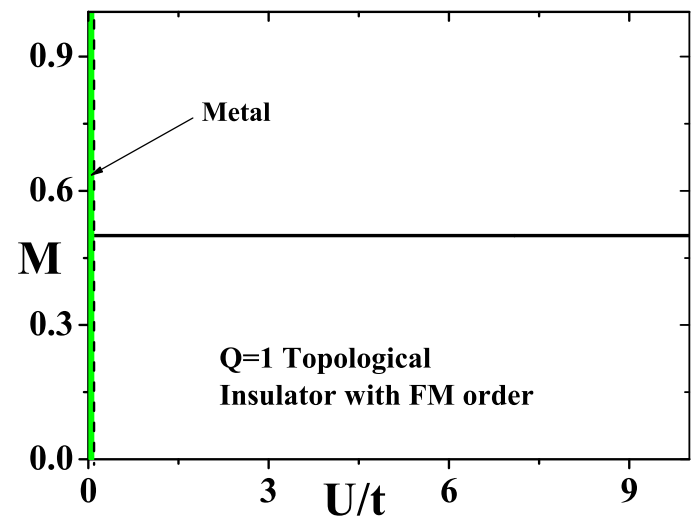

FIG. 12: (Color online) The staggered magnetization $M_{F}$ at the quarter filling $(d=0.5)$ for the case with the parameters $t^{\prime}=0.6 t, t^{\prime \prime}=-0.58 t$ and $\phi_{i j}=0.4 \pi$. There have two phases: the normal paramagnetic metal (the Green region) and the $Q=1$ topological insulator with FM order. In FM order, the ferromagnetization saturates suddenly.

\section{QUARTER FILLING CASE : $d=0.5$}

Secondly we consider the quarter filling case: $d=0.5$. In the global phase diagram in Fig.6, there exists an important line - the $d=0.5$ line. We found that at the quarter filling the ground state is a $Q=1$ topological insulator induced by the FM order $M_{F} \neq 0$. In Fig.6 we can see that there exist two phases divided by a quantum phase transition at $U / t=0.1$ for $d=0.5$ : the paramagnetic

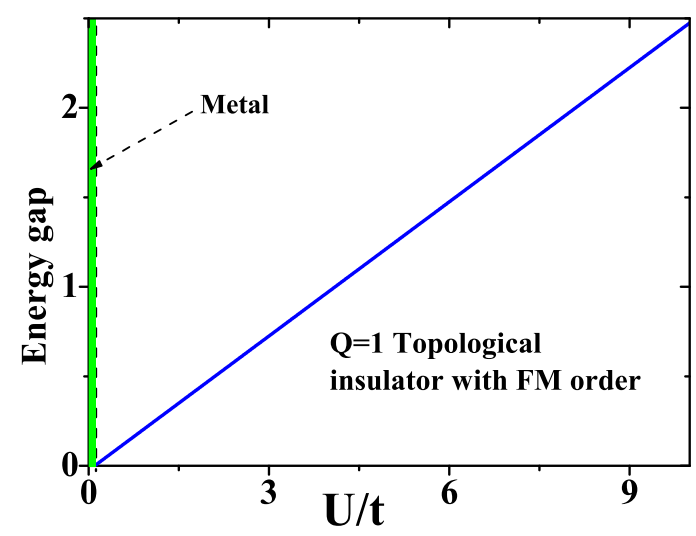

FIG. 13: (Color online) The electronic enegy gap at the quarter filling $(d=0.5)$ for the case with the parameters $t^{\prime}=0.6 t$, $t^{\prime \prime}=-0.58 t$ and $\phi_{i j}=0.4 \pi$. There have two phases: the normal paramagnetic metal (the Green region) and the $Q=1$ topological insulator with FM order.

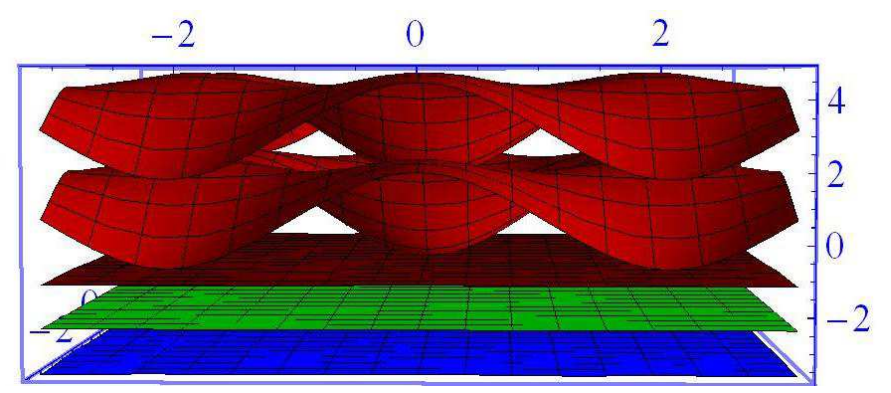

FIG. 14: (Color online) The electronic dispersion at the quarter filling $(d=0.5)$ for the case with the parameters $t^{\prime}=0.6 t$, $t^{\prime \prime}=-0.58 t$ and $\phi_{i j}=0.4 \pi, U / t=5.0$. The chemical potential $\mu_{\text {eff }}$ (the green plane) locates at the middle of the energy gap. The ground state is a $Q=1$ topological insulator with filled lowest topological flat band.

metallic state and the $Q=1$ topological insulator. In the weakly interacting case $(U / t<0.1)$, the ground state is paramagnetic metallic state with flat-band. Small interaction $(U / t>0.1)$ will drive the ground state to a $Q=1$ topological insulator with FM order. From this result one may guess that in flat-band limit (the flatness ratio is infinite), there exists only $Q=1$ topological insulator for $d=0.5$ and quantum phase transition between the paramagnetic metallic state and the $Q=1$ topological insulator occurs at $U / t=0^{+}$. This result is consistent to the rigorous proof in Ref. [13], in which it was proved that 


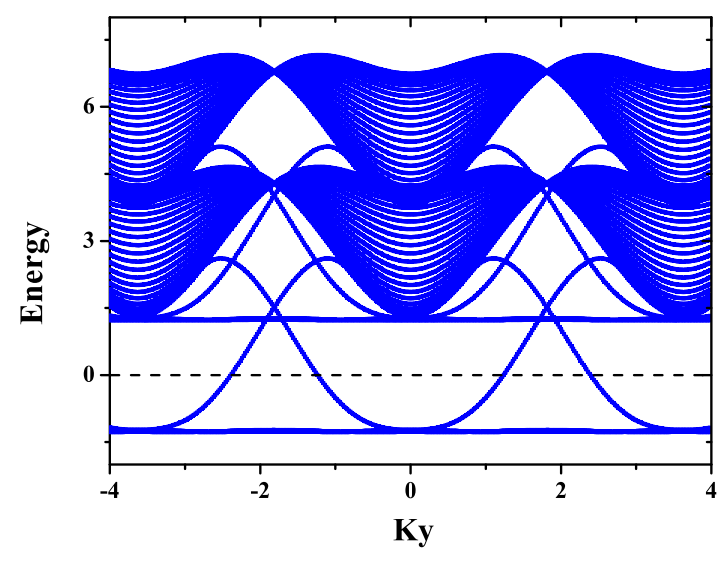

FIG. 15: (Color online) The Zigzag edge state along $\mathrm{x}$ direction at $U / t=5.0$ with $t^{\prime}=0.6 t, t^{\prime \prime}=-0.58 t, \phi_{i j}=0.4 \pi$ for the quarter filling case $d=0.5$. The black dash line is the position of chemical potential.

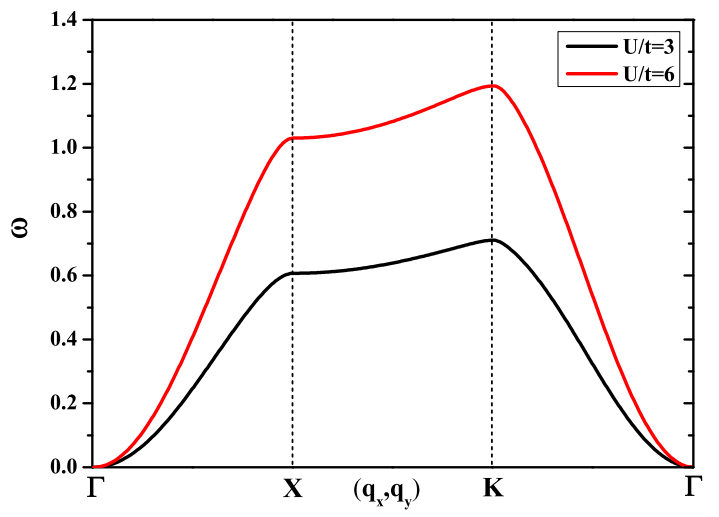

FIG. 16: (Color online) The dispersions of the spin wave of the FM order for the parameters are $t^{\prime}=0.6 t, t^{\prime \prime}=-0.58 t$, $\phi_{i j}=0.4 \pi$ with $U / t=3.0$ and $U / t=6.0$ at $d=0.5$.

the ground state of the Hubbard model with topological flat-bands is a ferromagnetic order when the lowest flat band is half-filled (that corresponds to the quarter filling case here).

In Fig.12 and Fig.13, we show the ferromagnetization $M_{F}$ and the energy gap for the case of $d=0.5$. In $Q=1$ topological insulator, the ferromagnetization suddenly jumps to 0.5 and gets saturated. In Fig.14, we plot the electronic dispersion of the case $d=0.5$ in $Q=1$ topological insulator region. From Fig.14, we can see clearly that the chemical potential $\mu_{\text {eff }}$ located at the middle of the energy gap, and the lowest band is filled, while other bands are empty. We also calculate the edge states of $Q=1$ topological insulator. Fig.15 illustrates the edge states of the topological state. This result con-

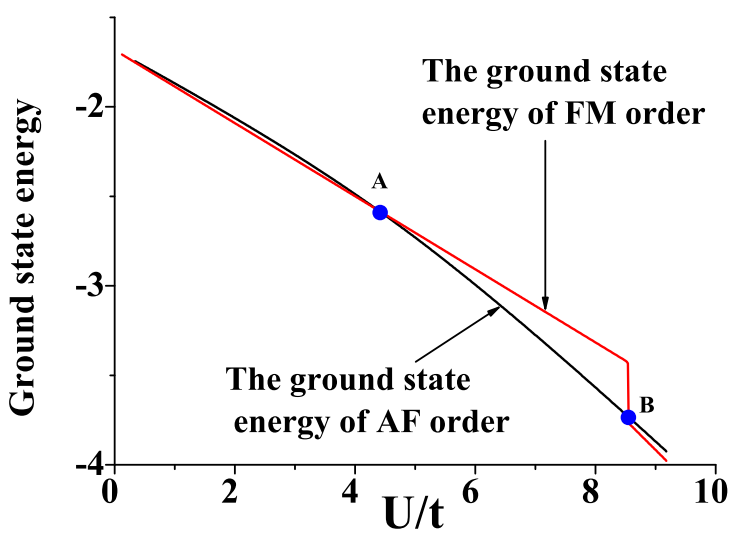

FIG. 17: (Color online) The ground state energies of AF order and FM order per site for the case of $d=0.3$ at $t^{\prime}=0.6 t$, $t^{\prime \prime}=-0.58 t$ and $\phi_{i j}=0.4 \pi$. The red line is the energy of FM order and the black line is the energy of AF order. The blue spots $A$ and $B$ denote the quantum phase transitions between $\mathrm{AF}$ order and FM order.

firms the topological property of this $Q=1$ topological insulator. In this phase, we also go beyond the mean field theory to study the collective spin fluctuations by RPA method (See Fig.16). In Fig.16, we can see that although the dispersion of electrons is flat, the collective spin modes are dispersive due the dispersive of the Matsubara Green's function (See the detailed calculation in Appendix). However, at low energy limit, the situation of FM order is much different from that of the half filling case of AF-SDW order parameter : around the $\Gamma$ point, the dispersion shows quadratic behavior instead of a linear one as $\omega(k) \propto q^{2}$.

\section{OTHER CASES}

Thirdly, we consider other cases, $d \neq 0, d \neq 0.5$. Here we take $d=0.3$ as an example.

From Fig.6, we can see that in the weak interaction case $(U / t<0.13)$, the ground state is normal metallic state with flat-band. Small interaction $(U / t>0.13)$ will drive the ground state to a FM metallic state. The phase transition between normal metal with $M_{F}=0$ and FMmetal with $M_{F} \neq 0$ is first order. The FM order parameter jumps up at $U / t=0.13$. When we increase interaction strength, the ground state energy for every site of the FM order is larger than AF order, the ground state becomes the AF order crossing a first order phase transition at $U / t=4.73$. An interesting property is that when we further increase interaction strength the ground state energy for every site of the FM order is lower than AF order again, the ground state re-enter the FM order crossing another first order phase transition at $U / t=8.55$. In Fig.17, we give the ground state energy comparation 


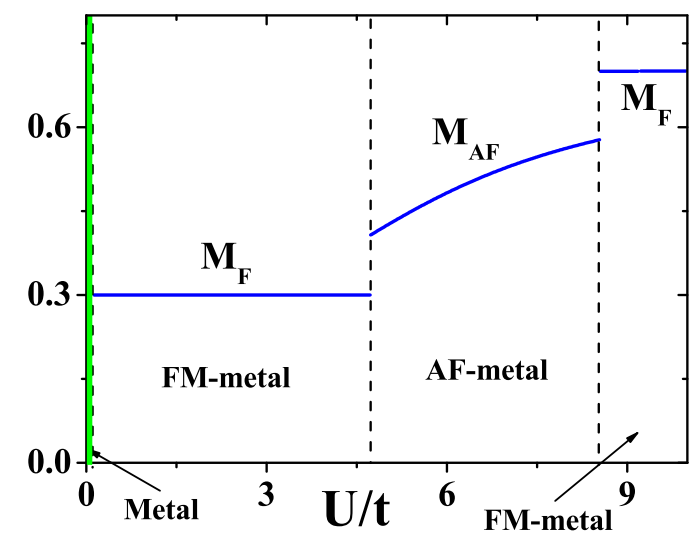

FIG. 18: ((Color online) The magnetization $\left(M_{F}\right.$ or $\left.M_{A F}\right)$ for the doping $d=0.3$ at $t^{\prime}=0.6 t, t^{\prime \prime}=-0.58 t$ and $\phi_{i j}=0.4 \pi$. There have three phases: paramagnetic Metal (the Green region), FM-metal and AF-metal.

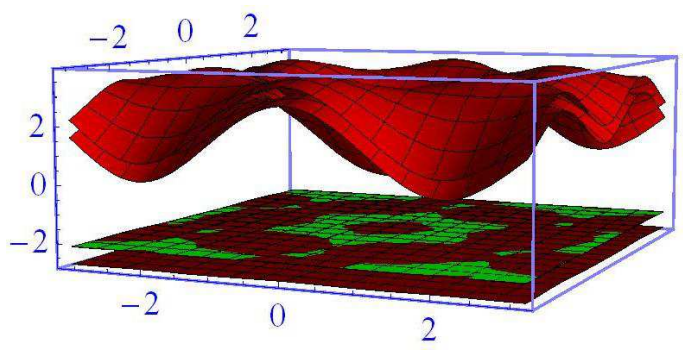

(a) $F M$ case: $U / t=2.0 \mathrm{~d}=0.3$

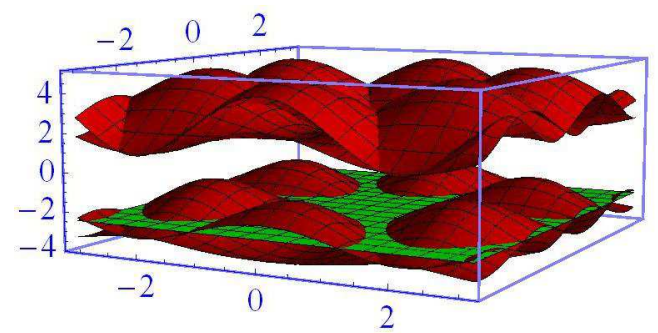

(b) AF case: $U / t=6.0 d=0.3$

FIG. 19: ((Color online) The electronic dispersion of the FM order and AF order at the doping $d=0.3$ for $t^{\prime}=0.6 t$, $t^{\prime \prime}=-0.58 t$ and $\phi_{i j}=0.4 \pi$. (a) is the FM case and (b) is the AF case, we can see clearly that $\mu_{e f f}$ (the green plane) locate in the spectrum.

between AF order and FM order per site at zero temperature when $d=0.3$, and we can see that there indeed have two transitions points $A$ and $B$. In Fig. 18 we show the magnetic order parameters. In Fig.19 we also plot the electronic dispersion at the same doping to compare the different dispersion in AF order and that in FM order. Correspondingly, the electronic band structure changes when phase transitions occur.

\section{CONCLUSION AND DISCUSSION}

In this paper, we investigated the magnetic properties (including ferromagnetism and antiferromagnetism) of a correlated topological insulator with flat band - the interacting spinful Haldane model by the mean field approach. We found that the AF order is more stable than FM order at (or near) half-filling due to energy gaining from super-exchange effect; while away from half-filling, FM order is more stable than AF order due to the flat band. In particular, at doping $d=0.5$, the system becomes a $Q=1$ topological insulator which is induced by the FM order. In addition, we found that in FM order the degenerate flat bands split without changing the flatness ratio, while in AF-SDW order, the electronic band structure changes totally and there doesn't exist flat-band at all. We also used the random-phase-approximation to calculate the dispersion of spin collective modes and found that in both FM order and AF order, the spin collective modes are dispersive. In AF order, the spin waves show linear dispersion while in FM order, the spin waves show quadratic dispersion.

\section{Acknowledgments}

This work is supported by NFSC Grant No. 11174035, National Basic Research Program of China (973 Program) under the grant No. 2011CB921803, 2012 CB921704.

\section{APPENDIX: RPA CALCULATIONS FOR SPIN COLLECTIVE MODES}

In this appendix, we use random-phase-approximation (RPA) to calculate the spin dispersion at doping $d=0$ and $d=0.5$. The spin excitations are obtained from the poles of the transverse spin susceptibility tensor, $\chi$, which is defined, in Matsubara form, as

$$
\chi_{i j}^{+-}\left(q, i \omega_{n}\right)=\int_{0}^{\beta} d \tau e^{i \omega_{n} \tau} \chi_{i j}^{+-}(q, \tau),
$$

where

$$
\chi_{i j}^{+-}(q, \tau)=\frac{1}{N_{s}}\left\langle T\left[\hat{S}_{i, q}^{+}(\tau), \hat{S}_{j,-q}^{-}(0)\right]\right\rangle
$$

and $i, j=a, b$ label the two sublattices and $S_{q}^{+}=$ $\sum_{k} c_{k+q, \uparrow}^{\dagger} c_{k, \downarrow}, S_{-q}^{-}=\sum_{k} c_{k-q, \downarrow}^{\dagger} c_{k, \uparrow}$ denote the spin operators for each sublattice. Using the Wick's theorem, we can derive the leading order spin suspectibility in the $\mathrm{AF}$ phase and FM phase as

$$
\begin{aligned}
\chi_{i j}^{+-(0)}\left(q, i \omega_{n}\right) & =-\frac{1}{\beta N_{s}} \sum_{k, \omega_{m}} G_{\uparrow}^{j i}\left(k, i \omega_{m}\right) \\
& \times G_{\downarrow}^{i j}\left(k-q, i \omega_{n}+i \omega_{m}\right) .
\end{aligned}
$$


In AF phase, the Matsubara Green's functions are :

$$
\begin{aligned}
& G_{\sigma}^{a a}(i \omega, k)=\sum_{j= \pm} \frac{\frac{1}{2}\left(1+\frac{t^{\prime} D+\sigma \Delta_{A}}{\xi_{\sigma, j}(k)}\right)}{i \omega-E_{\sigma, j}(k)}, \\
& G_{\sigma}^{b b}(i \omega, k)=\sum_{j= \pm} \frac{\frac{1}{2}\left(1-\frac{t^{\prime} D+\sigma \Delta_{A}}{\xi_{\sigma, j}(k)}\right)}{i \omega-E_{\sigma, j}(k)}, \\
& G_{\sigma}^{a b}(i \omega, k)=\sum_{j= \pm} \frac{\frac{\xi_{k}}{2 \xi_{\sigma, j}(k)}}{i \omega-E_{\sigma, j}(k)}, \\
& G_{\sigma}^{b a}(i \omega, k)=\sum_{j= \pm} \frac{\frac{\xi_{k}^{*}}{2 \xi_{\sigma, j}(k)}}{i \omega-E_{\sigma, j}(k)}
\end{aligned}
$$

where

$$
\begin{aligned}
E_{\sigma, \pm}(k) & = \pm \sqrt{\left(t A+t^{\prime \prime} E\right)^{2}+\left(t B+t^{\prime \prime} F\right)^{2}+\left(t^{\prime} D+\sigma \Delta_{A}\right)^{2}} \\
& -t^{\prime} C-\mu_{e f f}
\end{aligned}
$$$$
\xi_{\sigma, \pm}(k)= \pm \sqrt{\left(t A+t^{\prime \prime} E\right)^{2}+\left(t B+t^{\prime \prime} F\right)^{2}+\left(t^{\prime} D+\sigma \Delta_{A}\right)^{2}}
$$$$
\xi_{k}=-\left(t A+t^{\prime \prime} E\right)-i\left(t B+t^{\prime \prime} F\right)
$$

We may use the RPA approach to get the spin suscep- tibility tensor in AF order from the Dyson equation

$$
\begin{aligned}
\chi & =\chi^{0}+U \chi^{0} \chi \\
& \Rightarrow \chi=\left[\hat{I}-U \chi^{0}\right]^{-1} \chi^{0}
\end{aligned}
$$

where $\hat{I}$ denotes the $2 \times 2$ identity matrix. The poles of the spin susceptibility tensor, corresponding to the spin excitations, are then obtained from the condition :

$$
\operatorname{Det}\left[\hat{I}-U \chi^{0}\right]=0 \text {. }
$$

We note that the tensorial nature of the spin susceptibility is a consequence of two sublattices here.

The dispersion of spin collective modes with frequency $\omega$ and momentum $q$ is determined by above condition (22) after performing the analytic continuation $i \omega \rightarrow$ $\omega+i 0^{+}$. Finally the spin wave dispersion determinant is given by

$$
\begin{aligned}
D_{+-}(q, \omega) & =1-U \chi_{a a}^{+-(0)}-U \chi_{b b}^{+-(0)} \\
& +U^{2} \chi_{a a}^{+-(0)} \chi_{b b}^{+-(0)}-U^{2} \chi_{a b}^{+-(0)} \chi_{b a}^{+-(0)} \\
& =0
\end{aligned}
$$

where the elements of the spin susceptibility tensor $\chi^{+-(0)}$ are :

$$
\begin{aligned}
\chi_{a a}^{+-(0)}\left(q, \omega_{n}\right)= & -\frac{1}{N_{s}} \sum_{k}\left[\left(\frac{\left(1-\frac{t^{\prime} D(k)+\Delta_{A}}{\xi_{\uparrow}(k)}\right)\left(1+\frac{t^{\prime} D(k-q)-\Delta_{A}}{\xi_{\downarrow}(k-q)}\right)}{4\left(\omega_{n}-E_{\downarrow,+}(k-q)+E_{\uparrow,-}(k)\right)}+\frac{\left(1-\frac{t^{\prime} D(k)+\Delta_{A}}{\xi_{\uparrow}(k)}\right)\left(1-\frac{t^{\prime} D(k-q)-\Delta_{A}}{\xi_{\downarrow}(k-q)}\right)}{4\left(\omega_{n}-E_{\downarrow,-}(k-q)+E_{\uparrow,-}(k)\right)}\right) \theta\left(-E_{\uparrow,-}(k)\right)\right. \\
& \left.-\left(\frac{\left(1+\frac{t^{\prime} D(k)+\Delta_{A}}{\xi_{\uparrow}(k)}\right)\left(1-\frac{t^{\prime} D(k-q)-\Delta_{A}}{\xi_{\downarrow}(k-q)}\right)}{4\left(\omega_{n}-E_{\downarrow,-}(k-q)+E_{\uparrow,+}(k)\right)}+\frac{\left(1-\frac{t^{\prime} D(k)+\Delta_{A}}{\xi_{\uparrow}(k)}\right)\left(1-\frac{t^{\prime} D(k-q)-\Delta_{A}}{\xi_{\downarrow}(k-q)}\right)}{4\left(\omega_{n}-E_{\downarrow,-}(k-q)+E_{\uparrow,-}(k)\right)}\right) \theta\left(-E_{\downarrow,-}(k-q)\right)\right]
\end{aligned}
$$

$$
\begin{aligned}
\chi_{b b}^{+-(0)}\left(q, \omega_{n}\right)= & -\frac{1}{N_{s}} \sum_{k}\left[\left(\frac{\left(1+\frac{t^{\prime} D(k)+\Delta_{A}}{\xi_{\uparrow}(k)}\right)\left(1-\frac{t^{\prime} D(k-q)-\Delta_{A}}{\xi_{\downarrow}(k-q)}\right)}{4\left(\omega_{n}-E_{\downarrow,+}(k-q)+E_{\uparrow,-}(k)\right)}+\frac{\left(1+\frac{t^{\prime} D(k)+\Delta_{A}}{\xi_{\uparrow}(k)}\right)\left(1+\frac{t^{\prime} D(k-q)-\Delta_{A}}{\xi_{\downarrow}(k-q)}\right)}{4\left(\omega_{n}-E_{\downarrow,-}(k-q)+E_{\uparrow,-}(k)\right)}\right) \theta\left(-E_{\uparrow,-}(k)\right)\right. \\
& \left.-\left(\frac{\left(1-\frac{t^{\prime} D(k)+\Delta_{A}}{\xi_{\uparrow}(k)}\right)\left(1+\frac{t^{\prime} D(k-q)-\Delta_{A}}{\xi_{\downarrow}(k-q)}\right)}{4\left(\omega_{n}-E_{\downarrow,-}(k-q)+E_{\uparrow,+}(k)\right)}+\frac{\left(1+\frac{t^{\prime} D(k)+\Delta_{A}}{\xi_{\uparrow}(k)}\right)\left(1+\frac{t^{\prime} D(k-q)-\Delta_{A}}{\xi_{\downarrow}(k-q)}\right)}{4\left(\omega_{n}-E_{\downarrow,-}(k-q)+E_{\uparrow,-}(k)\right)}\right) \theta\left(-E_{\downarrow,-}(k-q)\right)\right]
\end{aligned}
$$




$$
\begin{aligned}
\chi_{b a}^{+-(0)}\left(q, \omega_{n}\right) & =-\frac{1}{N_{s}} \sum_{k} \frac{\xi_{k} \xi_{k-q}^{*}}{4 \xi_{\uparrow}(k) \xi_{\downarrow}(k-q)}\left[\left(\frac{1}{\omega_{n}-E_{\downarrow,-}(k-q)+E_{\uparrow,-}(k)}-\frac{1}{\omega_{n}-E_{\downarrow,+}(k-q)+E_{\uparrow,-}(k)}\right) \theta\left(-E_{\uparrow,-}(k)\right)\right. \\
& \left.-\left(\frac{1}{\omega_{n}-E_{\downarrow,-}(k-q)+E_{\uparrow,-}(k)}-\frac{1}{\omega_{n}-E_{\downarrow,-}(k-q)+E_{\uparrow,+}(k)}\right) \theta\left(-E_{\downarrow,-}(k-q)\right)\right]
\end{aligned}
$$

$$
\begin{aligned}
\chi_{a b}^{+-(0)}\left(q, \omega_{n}\right) & =-\frac{1}{N_{s}} \sum_{k} \frac{\xi_{k}^{*} \xi_{k-q}}{4 \xi_{\uparrow}(k) \xi_{\downarrow}(k-q)}\left[\left(\frac{1}{\omega_{n}-E_{\downarrow,-}(k-q)+E_{\uparrow,-}(k)}-\frac{1}{\omega_{n}-E_{\downarrow,+}(k-q)+E_{\uparrow,-}(k)}\right) \theta\left(-E_{\uparrow,-}(k)\right)\right. \\
& \left.-\left(\frac{1}{\omega_{n}-E_{\downarrow,-}(k-q)+E_{\uparrow,-}(k)}-\frac{1}{\omega_{n}-E_{\downarrow,-}(k-q)+E_{\uparrow,+}(k)}\right) \theta\left(-E_{\downarrow,-}(k-q)\right)\right]
\end{aligned}
$$

In FM order, we may use similar approach to calculate the spin suspectibility and the dispersion of spin collec- tive modes.
[1] X. G. Wen, Quantum Field Theory of Many-Body Systems, (Oxford Univ. Press, Oxford, 2004).

[2] K. V. Klitzing, G. Dorda, and M. Pepper, Phys. Rev. Lett. 45, 494 (1980).

[3] D. C. Tsui, H. L. Stormer, and A. C. Gossard, Phys. Rev. Lett. 48, 1559 (1982).

[4] F. D. M. Haldane, Phys. Rev. Lett. 61, 2015 (1988).

[5] E. Tang, J.-W. Mei, and X.-G. Wen, Phys. Rev. Lett. 106236802 (2011).

[6] K. Sun, Z. C. Gu, H. Katsura, and S. Das Sarma, Phys. Rev. Lett. 106, 236803 (2011).

[7] T. Neupert, L. Santos, C. Chamon, and C. Murdy, Phys. Rev. Lett. 106, 236804 (2011).

[8] D. N. Sheng, Z. C. Gu, K. Sun, and L. Sheng, Nature Commun. 2, 389 (2011).

[9] Yi Fei Wang, Zheng Cheng Gu, Chang De Gong, and D. N. Sheng, Phys. Rev. Lett. 107, 146803 (2011).
[10] A. Auerbach, Interacting Electrons and Quantum Magnetism (Springer-Verlag, New York, 1994).

[11] A. Mielke and H. Tasaki, Commun. Math Phys. 158, 341 (1993).

[12] H. Tasaki, Phy. Rev. Lett. 69, 1608 (1992).

[13] H. Katsura, I. Maruyama, A. Tanaka, and H. Tasaki, EPL, 91, 57007 (2010).

[14] A. Zhao and S. Q. Shen, Phys. Rev. B 85, 085209 (2012).

[15] J. He, S. P. Kou, Y. Liang, S. P. Feng, Phys. Rev. B 83, 205116 (2011).

[16] J. He, Y. H. Zong, S. P. Kou, Y. Liang, S. P. Feng, Phys. Rev. B 84, 035127 (2011).

[17] N .M .R. Peres, et al., Phys. Rev. B 70, 195122 (2004).

[18] G. Y. Sun and S. P. Kou, J. Phys.: Condens. Matter 23, 045603 (2011). 